\title{
Cost-effectiveness of neoadjuvant versus adjuvant chemotherapy for cT2-4N0-1 non-small cell lung cancer patients during initial treatment phase
}

\author{
Dongdong $\mathrm{Wu}^{1}$, Juan $\mathrm{Li}^{1}$, Yubo Wang ${ }^{1}$, Hao Huang ${ }^{1}$, and Chunji Huang ${ }^{1}$ \\ ${ }^{1}$ Third Military Medical University
}

November 11, 2020

\begin{abstract}
Objective: The choice between neoadjuvant chemotherapy (NAC) and adjuvant chemotherapy (AC) remains controversial in the treatment of non-small cell lung cancer (NSCLC). There is no significant difference in NAC and AC's effectiveness. We investigate the cost-effectiveness of NAC versus AC for NSCLC. Method: A decision tree model was designed from a payer perspective to compare NAC and AC treatments for NSCLC. Parameters included overall survival (OS), surgical complications, chemotherapy adverse event (AE), treatment initiation probability, treatment time frame, treatment cost, and quality of life (QOL). Sensitivity analyses were performed to characterize model uncertainty in the base cases. Result: With the same overall survival, AC treatment strategy produces a cost saving of $\mathrm{Y}=618.90$ and an incremental quality-adjusted life-years (QALY) of 0.10 years per patient. If median OS of NAC is 1.4 months more than AC, NAC would be cost effective (CE) at a $Y=35446 / Q A L Y$ threshold. The model was robust enough to handle variations to all input parameters except the overall survival. In the probability sensitivity analysis, AC remained dominant in $54.6 \%$ of simulations. Conclusion: The modeled cost effectiveness analysis indicates that with operable NSCLC, AC treatment is more cost effective compared to NAC. If NAC provides a longer survival advantage, this treatment strategy may be cost-effective. The OS is the main factor that influences cost-effectiveness analysis.
\end{abstract}

Cost-effectiveness of neoadjuvant versus adjuvant chemotherapy for cT2-4N0-1 non-small cell lung cancer patients during initial treatment phase

\section{Abstract}

Objective:The choice between neoadjuvant chemotherapy (NAC) and adjuvant chemotherapy (AC) remains controversial in the treatment of non-small cell lung cancer (NSCLC). There is no significant difference in NAC and AC's effectiveness. We investigate the cost-effectiveness of NAC versus AC for NSCLC.

Method: A decision tree model was designed from a payer perspective to compare NAC and AC treatments for NSCLC. Parameters included overall survival (OS), surgical complications, chemotherapy adverse event (AE), treatment initiation probability, treatment time frame, treatment cost, and quality of life (QOL). Sensitivity analyses were performed to characterize model uncertainty in the base cases.

Result: With the same overall survival, AC treatment strategy produces a cost saving of $\mathrm{Y}=618.90$ and an incremental quality-adjusted life-years (QALY) of 0.10 years per patient. If median OS of NAC is 1.4 months more than AC, NAC would be cost effective (CE) at a Y=35446/QALY threshold. The model was robust enough to handle variations to all input parameters except the overall survival. In the probability sensitivity analysis, AC remained dominant in $54.6 \%$ of simulations.

Conclusion: The modeled cost effectiveness analysis indicates that with operable NSCLC, AC treatment is 
more cost effective compared to NAC. If NAC provides a longer survival advantage, this treatment strategy may be cost-effective. The OS is the main factor that influences cost-effectiveness analysis.

Key words: cost-effectiveness, neoadjuvant chemotherapy, adjuvant chemotherapy, non-small cell lung cancer

\section{Introduction}

Non-small cell lung cancer (NSCLC) is a frequent malignancy and the most common cause of cancer-related death among males and females in the world, resulting in a large social and economic burden [1]. The National Comprehensive Cancer Network (NCCN) guidelines recommended surgery followed by adjuvant chemotherapy (AC) for cT2-4N0-1 NSCLC with a supplementary instruction that neoadjuvant chemotherapy (NAC) followed by surgery should also be considered for these patients [2]. The use of adjuvant chemotherapy for surgically resectable NSCLC has been well established by several randomized trials and meta-analysis which demonstrated clear survival benefits over surgery alone [3]-[6].

Despite similar overall survival (OS) and disease-free survival (DFS), evidence is not very robust with regard to the benefits of NAC. The original purposes of administering chemotherapy before surgery included: improving operability by reducing tumor tissue size, increasing the likelihood of administering the maximal planned dose of chemotherapy, and reducing the likelihood of micro-metastasis and uncomplete resection (R1/R2) [7][8]. At the same time, there were several potential risks of NAC including delaying operation, increasing the postoperative complication for the postoperative toxicity, and making the tumor unresectable [9]. Thus, there is widespread debate in the use of NAC and adjuvant chemotherapy (AC) with NSCLC patients.

The comparative effectiveness of NAC versus AC in terms of DFS and OS remains controversial. The study by Brandt et al. aims to evaluate whether treatment strategy of NAC or AC is better for cT2-4N0-1 NSCLC patients through a propensity score match analysis [10]. They analyzed 92 matched-pair patients and ultimately demonstrated that there was no significant difference in DFS and OS between treatment cohorts. Previous investigators have tried to answer this question. The NATCH trial recruited 624 patients with stage I-IIIA, N0-N1 NSCLC to compare the effect of tree therapeutic strategies (NAC, AC and surgery alone) [3]. The three arms trial found that there was no significant difference in DFS and OS between those treatments. The open label randomized trial by Westeel et al. showed the same conclusion in early stage NSCLC patients [11]. Coincidentally, the meta-analysis of trials also did not demonstrate differences in OS and DFS between NAC and AC [12][13].

Although they show similar clinical outcomes, most of the previous literature supports the use of adjuvant chemotherapy over neoadjuvant chemotherapy. However, there are some theoretical difference that have not been specifically addressed or adequately studied. Firstly, administration of NAC could have the potential to reduce tumor size before surgery and increase the complete resection rate [9]. Secondly, the patients who received NAC might have higher surgery complications and mortality rate, due to the preoperative chemotherapy toxicity [7]. Thirdly, patients who received NAC had better chemotherapy tolerance than the patients who underwent $\mathrm{AC}[3][10]$.

These theoretical differences of AC and NAC may impact the cost associated with caring for NSCLC patients. What's more, under the circumstances, there was no robust evidence on the outcomes of NAC versus AC in terms of OS and DFS. Cost-effectiveness analyses may contribute to decision-making among NSCLC patients for whom the optimal therapeutic regimen is unclear. Until now, cost-effectiveness research comparing the NAC and AC treatment protocols in lung cancer, was absent. The previous cost-effectiveness studies about neoadjuvant chemotherapy versus adjuvant chemotherapy, focused only on ovarian cancer and head neck cancer [14]-[19]. Therefore, we compared the cost effectiveness of AC and NAC treatment strategies in the case of NSCLC, through a decision tree-modeled cost effectiveness analysis from the perspective of the payer.

\section{Method}

\subsection{Model Structure}


We created a decision tree model using the software package Tree-Age pro 2011 to compare the health and economic impact of NAC and AC for cT2-4N0-1 NSCLC patients from the payer's perspective. Costs were measured using a China Medicare care perspective, and outcome of patients include OS, quality-adjusted life-years (QALYs), health utilities value and treatment time. The utility of disease was calculated according to published utilities. The event outcome was informed by the study of Brandt et al.

In the model, patients received either surgery followed by four rounds of adjuvant chemotherapy, or 2 rounds of neoadjuvant chemotherapy followed by lung surgery and an additional 2 rounds of adjuvant chemotherapy. We assumed that the chemotherapy regimen was intravenous paclitaxel/carboplatin, for the previous study had showed there was no significant association of chemotherapy regimens for NAC and AC and there was no significant difference in the survival for different chemotherapy regimens [10]. For every set of chemotherapy cycles, patients could experience grade 3 or 4 chemotherapy-related adverse events (AE). Meanwhile, during the surgery (both NAC and $\mathrm{AC}$ ), the patient also could experience surgery complications. If patients experienced adverse event (AE) or complications from chemotherapy or surgery respectively, they could either recover or die from the event (Fig.1). The goals of our study were to compare the cost-effectiveness in the initial stage and the treatment stage. Cost and prognosis for patients treated with NAC and AC during the progressive stage were not included in the model.

\subsection{Effectiveness and quality of life}

Based on the study by Brandt et al. there was no significant difference in median OS between NAC and AC. So, we assumed that the OS (9.1 years) and quality of life (QOL) during the progression-free survival stage were equal. The parameters like probabilities of $\mathrm{AE}$ and complications, are also based on the published results in this study, which are listed in Table 1. As data for postoperative death couldn't be sourced, 30-day mortality and 90-day mortality information was integrated into postoperative complication mortality. Based on this study, the patients treated with neoadjuvant chemotherapy all received the lung cancer surgery, and the patients treated with surgery as the initial therapy, all received the adjuvant chemotherapy. In considering chemotherapy tolerance, we take the proportion of grade 3 and $4 \mathrm{AE}$ as the input parameter. There were no deaths for chemotherapy and AE.

Quality of life was estimated using standard health utility weights. The utility weights of stable disease and progressive-free disease were calculated according to published study[22][24]. As there is a shortage of quality of life studies in the case of lung cancer surgery complications, we use the utility value of pneumothorax in the study of Handorf et al [24] as the 3 and 4 grade complication utility weights. The average utility weight of every treatment procedure was shown in 2 , and the time frames of each treatment are based on the study of Dendulur et al [21] and Lugg et al [25]. In the model, the treatment of patients with 3 or 4 grade AE will add 0.73 months to basic time of surgery and chemotherapy, while treatment of those with 3 or 4 grade complication will add 0.5 months. The utility weight of patients with 3 or 4 grade AE and complication were 0.45 . The utility weight during the time 6 months after initial treatment remained unchanged.

\subsection{Cost}

Because the study is about the treatment strategies in the initial treatment phase, costs associated with the treatment of recurrence and rehabilitation were assumed to be equivalent for NAC and AC. We also assumed that the two treatment strategies have the same costs in biochemical testing, imaging examination, pathological examination, venipuncture related protocols and diagnosis. Cost calculation was only done for medical related items that might make a big difference in NAC and $\mathrm{AC}$ treatments.

Costs associated with (medical) caring for NSCLC patients included the cost of surgery procedure, major late complications, chemotherapy administration and chemotherapy adverse events (Table 3). The final cost of the surgery was calculated as the sum of base surgical procedures cost, additional surgical procedures cost and hospitalization costs (including the cost of surgery complication). The surgery (pneumonectomy, bi-lobectomy, lobectomy, or segmentectomy) costs were constructed from Medical programs fee schedule (MPFS) [29]. The chemotherapy costs were measured the costs of main chemotherapy drugs (paclitaxel and carboplatin). Based on recommendations in the current guidelines, one cycle chemotherapy dosing was 
calculated as $200 \mathrm{mg} / \mathrm{m}^{2}$ for paclitaxel and an area under the curve of 6 for carboplatin, [2].

The major high-grade chemotherapy adverse events were neutropenia, thrombocytopenia, anemia, nausea \& vomiting, febrile neutropenia, diarrhea, hyperglycemia, arthralgias, myalgias, fatigue, sensory neuropathy, allergic reaction [3]. The definition of $\mathrm{AE}$ grade followed that of the Common Terminology Criteria for Adverse Events (CTCAE) Version 5.0 [27] and Guideline of Chinese Society Oncology (CSCO) Primary Lung Cancer [28]. The costs of AE were estimated by the core medical care components, empirical data, and expert opinion on implementation strategies.

All cost estimates were calculated in 2020 RMB with the medical care components of Consumer Price Index from the price dataset in Shanghai Price Control Administration [29].

\subsection{Sensitivity analyses}

To address the uncertainty of costs and outcomes in our model, we did several 1-way sensitivity analyses. The parameters listed in Table 1-3 were varied in specified ranges of NAC and AC. In the base case analysis, a chance of $\pm 20 \%$ to the original values were set as the upper and lower bounds.

We also performed probabilistic sensitivity analysis (PSA) to assess the combined effect of multiple parameter uncertainty on the increment cost effectiveness ratio (ICER.) Factors varied in sensitivity analyses included OS, base-case event probabilities, costs, utility weights and treatment time frames with distributions selected based on published literature [30]. Costs and treatment time obeyed gamma distribution, with a standard error of $10 \%$ of the original value. Utilities distribution reflected a triangular distribution, and base-case utility weights were set as the most likely value with minimum and maximum values of \pm 0.05 . Events probabilities distribution were beta distribution, with alpha and beta parameters calculated through the event's incidence in the study of Brandt et al [10]. The PSA was performed with 10,000 Monte Carlo simulations across all distributions.

\section{Result}

\subsection{Base case}

In this model AC is the dominant strategy with the ICRE of 6384.14 RMB/QALY. Using the base case probabilities and cost, $\mathrm{AC}$ cost is $105,453.73 \mathrm{RMB}$ per patient which is $618.90 \mathrm{RMB}$ fewer than NAC cost (with 106,072.63 per patients) in the initial treatment phase. And the average QALY is 8.66 years for AC, and 8.56 for NAC.

\subsection{Sensitivity Analyses}

We performed a series of one-way sensitivity analyses to discover the effect of the different parameter's uncertainty on the ICER. The Tornado diagram shows the effect of those parameters that led to a change in the ICER from big to small (Fig.2). In our model, the ICER was most sensitive to variations in OS of NAC and AC and, when the medial OS of NAC is 0.12 years more than AC, the NAC will be cost-effective at the 35,446 RMB threshold (Fig.3). The sensitivity analyses suggest that our model was robust enough to handle to the uncertainty surrounding the parameters of cost, base case probabilities, treatment time and utility weight.

In PSA, AC was the dominant strategy in $64.3 \%$ of 10,000 Monte Carlo simulations and, at the threshold of 35,446 RMB per QALY, there is a 54.1\% probability that AC strategy is cost-effective. Fig.4 shows the corresponding cost effectiveness acceptability curve for NAC and AC. Therefore, the statement about basic simulation regarding incremental costs, effectiveness and the ICER, is stable.

\section{Discussion}

At present, the cost comparative between NAC and AC for lung cancer patients has not been extensively studied. We searched the PubMed database to identify cost-effectiveness analyses of NAC and AC in NSCLC, published before January 2020. Tree searches were done, with the following search terms: adjuvant chemotherapy and neoadjuvant chemotherapy, cost effectiveness; cost effectiveness, adjuvant chemotherapy, 
neoadjuvant chemotherapy and lung cancer; cost effectiveness, preoperative, postoperative, chemotherapy and lung cancer. There are 7 studies that had evaluated the cost-effectiveness of NAC and AC. But none of the studies had assessed the cost-effectiveness specific to lung cancer. Off the 7 cases mentioned, 6 are about ovarian cancer and 1 is about head and neck cancer [14]-[19].

Among those research studies, the study in head and neck cancer showed that NAC is more cost-effective than AC [19]. 4 of the studies[14]-[17] about ovarian cancer showed the same result and two studies [18][19] showed that AC as being dominated strategy. Findings from the previous research studies (which stated that the therapeutic regimen is more cost-effective) do not remain constant. Our study showed that NAC is more cost-effective than AC, with a cost saving of $Y=618.90$ and a QALY increment of 0.10 years per patients.

In contrast to those previous studies, the input parameters in our model, included the cost of chemotherapy adverse events, Among those research studies, only one [14] explicitly incorporated the chemotherapy adverse event into the model. The reason for this maybe that there were no significant differences in the chemotherapy-related toxicities for NAC and AC in ovarian cancer and head \& neck cancer [15][31]. For NSCLC patients however, the tolerability of chemotherapy and the ratio of AE are significantly different in $\mathrm{NAC}$ and AC as supported by the NATCH phase 3 trial [3] and the study by Brant et al [10]. Nonetheless, the difference in tolerability of chemotherapy and the ratio of AE does not contribute to the OS. Besides this, the treatment expense of 3 and 4 grade $\mathrm{AE}$ is even higher than the surgery procedure cost [14]. Thus, although the result was not sensitive to the ratio and cost of $\mathrm{AE}$ in our model, we think cost comparison between NAC and $\mathrm{AC}$ needs to consider the impact of $\mathrm{AE}$.

In our study, the sample population is cT2-4N0-1 NSCLC patients, excluding stage IV patients (for whom NCCN guidelines recommend two treatment strategies). The choice of NAC and AC is a tough one in the initial treatment phase. The patients who are less clinically at-risk, benefit more from AC, while the stage IV patients are recommended systemic therapy by NCCN and there is robust evidence in support of the same [2]. Thus, our study focused on the sample population of patients whose treatment strategies were controversial.

However, most studies compared NAC or AC with the treatment of surgery alone, and estimated survival benefit. Very few studies directly compared the two chemotherapy approaches [7][8]. The head to head comparison of the studies of NATCH and Brandt et al in light of NAC and AC, showed that there are no statistically significant differences in the OS and DFS. But, the NATCH trial was criticized for being overly optimistic and over representing the study design [7][8]. The percentage of stage I disease patients who did not benefit from chemotherapy in the study cohort is $75 \%$. In comparison with the meta-analysis [12], the stage I disease patients in NAC cohorts account for nearly 50\%. This is the reason base case probabilities are based on the study of Brandt et al in our model.

Beside this, our study was based on real-world data. The study generated two groups (92 in NAC and 92 in $\mathrm{AC}$ ) with comparable characteristics through strict exclusion criteria and propensity score matching analyses, to prevent selection bias related to nonrandomized cohort. The ratio of males and females more closely reflects the real-word population of NSCLC patients who need to receive either NAC or AC.

What's more, the study sample population excluded the patients with microscopic and macroscopic residual disease (R1/R2 resection), which avoids the influence of surgery discrepancy (since the surgery which results in resection to minimal or no gross residual disease may be associated with a long-term survival advantage). The single-center data source reduced the effectiveness of surgery.

There are some limitations to our model. As with all cost-effectiveness analyses, assumptions in clinical base cases, cost and quality of life are important to the projected outcomes determined by the model. Consequently, 1-way and probability sensitivity analyses were performed to test our assumptions. The sensitivity analyses showed that our model was robust enough to handle to the variation of cost, quality of life, ratio of complication and AE. But the variation of OS would change the conclusion of the costeffectiveness analysis in our model. 
The medial OS is the most sensitive parameter in our cost-effectiveness analysis model. The study of Brandt et al, NATCH trial and Tim et al all show that the medial OS of NAC and AC have no significant difference[3][10][12][13]. In fact, the difference ( $<0.12$ years) of NAC's and AC's medial OS (9.22 VS 8.98 year in Brandt et al) is enough to change the conclusion of our model. Using 9.22 and 8.98 years as the OS of NAC and AC in our model, NAC is more cost effective with the ICRE of 3070 RMB/QALY. Given the concern of survival in lung cancer treatment for NSCLC patients, it is important to evaluate sensitivity of OS in cost-effectiveness analysis.

Simultaneously, there are several assumptions in the cost. To make the model clear and accurate, our cost measures were intentionally confined to the associated costs of initial treatment phase. This was also based on the assumption that there would not be a significant difference between treatment and ongoing care in the NAC and AC groups beyond the initial recovery period. However, if long term surgery complication or chemotherapy adverse events affected one group and increased the follow-up medical treatment, the difference of NAC and AC cost may be improperly over underestimated. Beside this, patients need to do more imaging examination in the NAC treatment, and one patient did not only have once AE in the chemotherapy treatment.

In addition, probabilities used in estimating surgery complication and postoperative death, may be overrated in NAC, because patients with more comorbidities or more complex diseases may be more likely to receive NAC. That is why the ratio of related complication in NAC is higher than AC in our model. In the NATCH trial however, the postoperative death of AC is higher than NAC (5\% VS 7.5\%) and the ratio of complication in the multicenter randomized controlled trial (CRT) is influenced by the level of the surgery team. In the case of chemotherapy tolerance, our model did not consider the probability of completed chemotherapy (full dose and full cycles). The chemotherapy adverse events of NAC and AC had no significate difference (25.4\% VS $27.3 \%$ ) in the NATCH trial. Thus, the base case probability may change in the future with more and more comparative research conducted about the NAC and AC.

Currently, there are no comparative studies that examine of quality of life in NAC and AC for NSCLC patients. Hence, we assumed that the health utility weight of NAC and AC is the same in various treatment stages. We also used health utility weights from previously published literature with NSCLC treatment phase related utilities used whenever possible. There is also a difference in psychological effects after neoadjuvant chemotherapy and primary surgery.

\section{Conclusion}

Despite the higher levels of chemotherapy tolerance and the same survival rate in NAC, AC has a favorable cost-effectiveness profile in the NSCLC initial treatment phase. The cost-effectiveness analysis is sensitive to survival at classic WTP thresholds. Based on the current evidence, the NAC as a general ffirst choice treatment in cT2-4N0-1 NSCLC patients is not supported. To better assess the relative merits of these therapeutic regimens, attention should not only be given to OS and DFS, but also to the QOL and costeffectiveness, especially when the number of lung cancer patients and treatment burden increase greatly. In view of the insufficiency in head to head trials and clinically enriched dataset, the cost-effectiveness analysis of NAC and AC can benefit from more cohort studies.

\section{References}

1. Bray, F., Ferlay, J., Soerjomataram, I., Siegel, R. L., Torre, L. A., \& Jemal, A. Global cancer statistics 2018: GLOBOCAN estimates of incidence and mortality worldwide for 36 cancers in 185 countries. CA: A Cancer Journal for Clinicians.2018; 68(6):394-424.

2. National Comprehensive Cancer Network. Clinical Practice Guidelines in Oncology (NCCN guidelines): Non-small cell lung cancer. Version 6. 8 17. https://www.nccn.org/professionals/physician_gls/default.aspx. Accessed 5 July 2020.

3. Felip E, Rosell R, Maestre JA, et al. Preoperative chemotherapy plus surgery versus surgery plus adjuvant chemotherapy versus surgery alone in early-stage non- small-cell lung cancer. J Clin Oncol 2010;28:3138-45. 
4. Berry MF, Coleman BK, Curtis LH, Worni M, D'Amico TA, Akushevich I. Berry MF, Coleman BK, Curtis LH, Worni M, D'Amico TA, Akushevich I. Benefit of adjuvant chemotherapy after resection of stage II (T1-2N1M0) non-small cell lung cancer in elderly patients. Ann Surg Oncol. 2015;22(2):642648.

5. Pignon JP, Tribodet H, Scagliotti GV, et al. Lung adjuvant cisplatin evaluation: a pooled analysis by the LACE Collaborative Group. J Clin Oncol. 2008;26(21):3552-3559.

6. Arriagada R, Bergman B, Dunant A, et al. Cisplatin-based adjuvant chemotherapy in patients with completely resected non-small-cell lung cancer. N Engl J Med. 2004;350(4):351-360.

7. Pu, C. Y., \& Yendamuri, S. Neoadjuvant versus adjuvant chemotherapy for resectable non-small cell lung cancer debate revisited. Journal of Thoracic Disease. 2019;11(12):5646-5648.

8. Subramanian, M. P., \& Puri, V. Neoadjuvant vs. adjuvant chemotherapy in locally advanced non-small cell lung cancer-is timing everything? Journal of Thoracic Disease. 2019;11(12): 5674-5676.

9. NSCLC Meta-analysis Collaborative Group. Preoperative chemotherapy for non-small-cell lung cancer: a systematic review and meta-analysis of individual participant data. Lancet, 2014;383:1561-71.

10. Brandt, W. S., Yan, W., Zhou, J., Tan, K. S., Montecalvo, J., Park, B. J., .. Jones, D. R. Outcomes Following Neoadjuvant or Adjuvant Chemotherapy for cT2-4N0-1 Non-Small Cell Lung Cancer: A Propensity-Matched Analysis. J Thorac Cardiovasc Surg. 2020;157(2):743-753.

11. Westeel, V., Quoix, E., Puyraveau, M., Lavole, A., Braun, D., Laporte, S., Milleron, B. A randomised trial comparing preoperative to perioperative chemotherapy in early-stage non-small-cell lung cancer (IFCT 0002 trial). European Journal of Cancer. 2013;49(12):2654-2664.

12. Lim E, Harris G, Patel A, Adachi I, Edmonds L, Song F. Preoperative versus postoperative chemotherapy in patients with resectable non-small cell lung cancer: systematic review and indirect comparison meta-analysis of randomized trials. J Thorac Oncol. 2009;4(11):1380-1388.

13. Zhang XN, Huang L. Neoadjuvant chemotherapy followed by surgery versus upfront surgery in nonmetastatic non-small cell lung cancer: systematic review and meta-analysis of randomized controlled trials. Oncotarget. 2017;8(52):90327-90337.

14. Tran AQ, Erim DO, Sullivan SA, et al. Cost effectiveness of neoadjuvant chemotherapy followed by interval cytoreductive surgery versus primary cytoreductive surgery for patients with advanced stage ovarian cancer during the initial treatment phase. Gynecol Oncol. 2018;148(2):329-335.

15. Cole, A. L., Barber, E. L., Gogate, A., Tran, A. Q., \& Wheeler, S. B. Economic Analysis of Neoadjuvant Chemotherapy Versus Primary Debulking Surgery for Advanced Epithelial Ovarian Cancer Using an Aggressive Surgical Paradigm. International Journal of Gynecological Cancer. 2018; 28(6): 1077-1084.

16. Rowland, M. R., Lesnock, J. L., Farris, C., Kelley, J. L., \& Krivak, T. C. Cost-utility comparison of neoadjuvant chemotherapy versus primary debulking surgery for treatment of advanced-stage ovarian cancer in patients 65 years old or older. American Journal of Obstetrics and Gynecology. 2015; 212(6):763.e1-763.e8.

17. Poonawalla, I. B., Lairson, D. R., Chan, W., Piller, L. B., \& Du, X. L. Cost-Effectiveness of Neoadjuvant Chemotherapy versus Primary Surgery in Elderly Patients with Advanced Ovarian Cancer. Value in Health. 2015; 18(4):387-395.

18. Onisim, A., Achimas-Cadariu, P., Iancu, M., Puscas, E., Bodog, A., \& Vlad, C. Clinical outcomes and cost-effectiveness of primary treatment of ovarian cancer in North-Western Romania. Journal of BUON. 2019; 24(3):982-990.

19. Forde GK, Chang J, Ziogas A. Cost-effectiveness of primary debulking surgery when compared to neoadjuvant chemotherapy in the management of stage IIIC and IV epithelial ovarian cancer. Clinicoecon Outcomes Res. 2016;8:397-406.

20. Himmel, M., Hartmann, M., \& Guntinas-Lichius, O. Cost effectiveness of neoadjuvant chemotherapy in locally advanced operable head and neck cancer followed by surgery and postoperative radiotherapy: A Markov model-based decision analysis. Oncology (Switzerland). 2013;84(6):336-341.

21. Denduluri, N., Patt, D. A., Wang, Y., Bhor, M., Li, X., Favret, A. M., Lyman, G. H. (2015). Dose delays, dose reductions, and relative dose intensity in patients with cancer who received adjuvant or neoadjuvant chemotherapy in community oncology practices. JNCCN Journal of the National 
Comprehensive Cancer Network. 2015; 13(11):1383-1393.

22. Grutters, J. P. C., Joore, M. A., Wiegman, E. M., Langendijk, J. A., De Ruysscher, D., Hochstenbag, M., Pijls-Johannesma M. (2010). Health-related quality of life in patients surviving non-small cell lung cancer. Thorax. 2010;65(10):903-907.

23. Paracha N, Abdulla A, MacGilchrist KS. Systematic review of health state utility values in metastatic non-small cell lung cancer with a focus on previously treated patients. Health Qual Life Outcomes. 2018;16(1):179.

24. Handorf, E. A., McElligott, S., Vachani, A., Langer, C. J., Bristol Demeter, M., Armstrong, K., \& Asch, D. A. Cost Effectiveness of Personalized Therapy for First-Line Treatment of Stage IV and Recurrent Incurable Adenocarcinoma of the Lung. Journal of Oncology Practice. 2012;8(5):267-274.

25. Lugg, S. T., Agostini, P. J., Tikka, T., Kerr, A., Adams, K., Bishay, E., Naidu, B. Long-term impact of developing a postoperative pulmonary complication after lung surgery. Thorax. 2016;71(2):171-176.

26. Xin, L., Jufang, S., Jian, Y., \& Wanqing, C. Influencing Factors of Hospitalization Expenses of Surgical Lung Cancer Patients:An Analysis of 5222 Cases. China Cancer. 2019:28(12):922-926.

27. National Institutes of Health, National Cancer Institute: Common Terminology Criteria for Adverse Events (CTCAE). https://ctep.cancer.gov/protocolDevelopment/electronic_applications/ctc.htm\#ctc_50. Accessed 5 July 2020.

28. Chinese Society Oncology Cancer (CSCO). Guideline of Chinese Society Oncology Cancer (CSCO) Primary Lung Cancer. People's Medical Publishing House; 2019.

29. Shanghai municipal health commission. Medical programs fee schedule (MPFS). http://wsjkw.sh.gov.cn/ylsfbz/index.html. 2017. Accessed 5 July 2020.

30. Briggs, AH., Claxton, K., Sculpher, MJ. Decision modelling for health economic evaluation. Oxford University Press; 2006.

31. Fagotti A, Ferrandina G, Vizzielli G, Fanfani F, Gallotta V, Chiantera V, et al. Phase III randomised clinical trial comparing primary surgery versus neoadjuvant chemotherapy in advanced epithelial ovarian cancer with high tumor load (SCORPION trial): Final analysis of peri-operative outcome. Eur J Cancer. 2016; 59:22-33.

\section{Hosted file}

Unblinded Title Page.pdf available at https://authorea.com/users/374783/articles/492221cost-effectiveness-of-neoadjuvant-versus-adjuvant-chemotherapy-for-ct2-4n0-1-non-smallcell-lung-cancer-patients-during-initial-treatment-phase

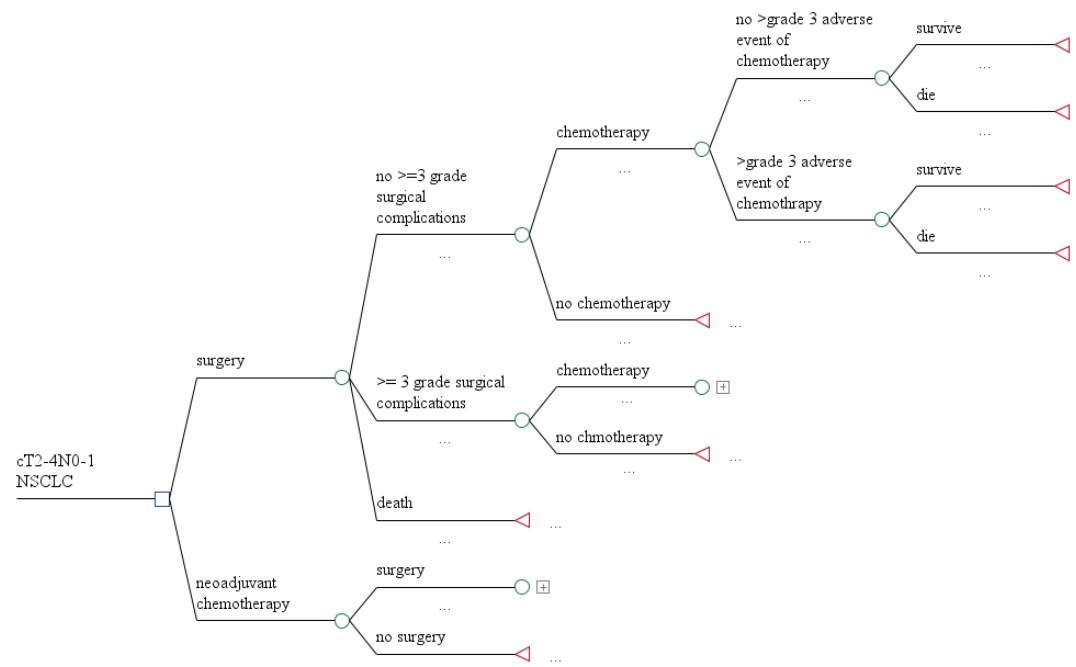



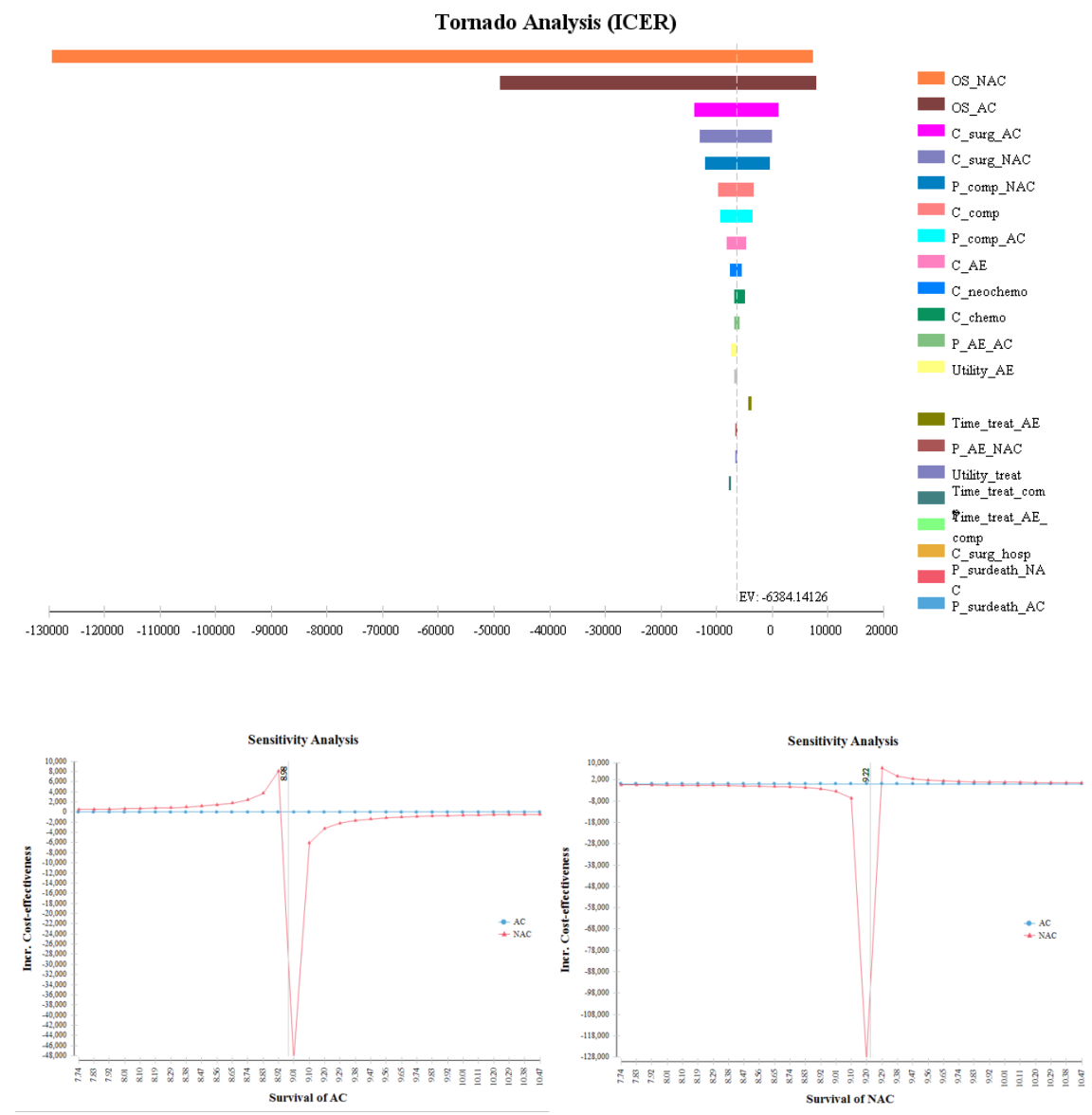

CE Acceptability Curve

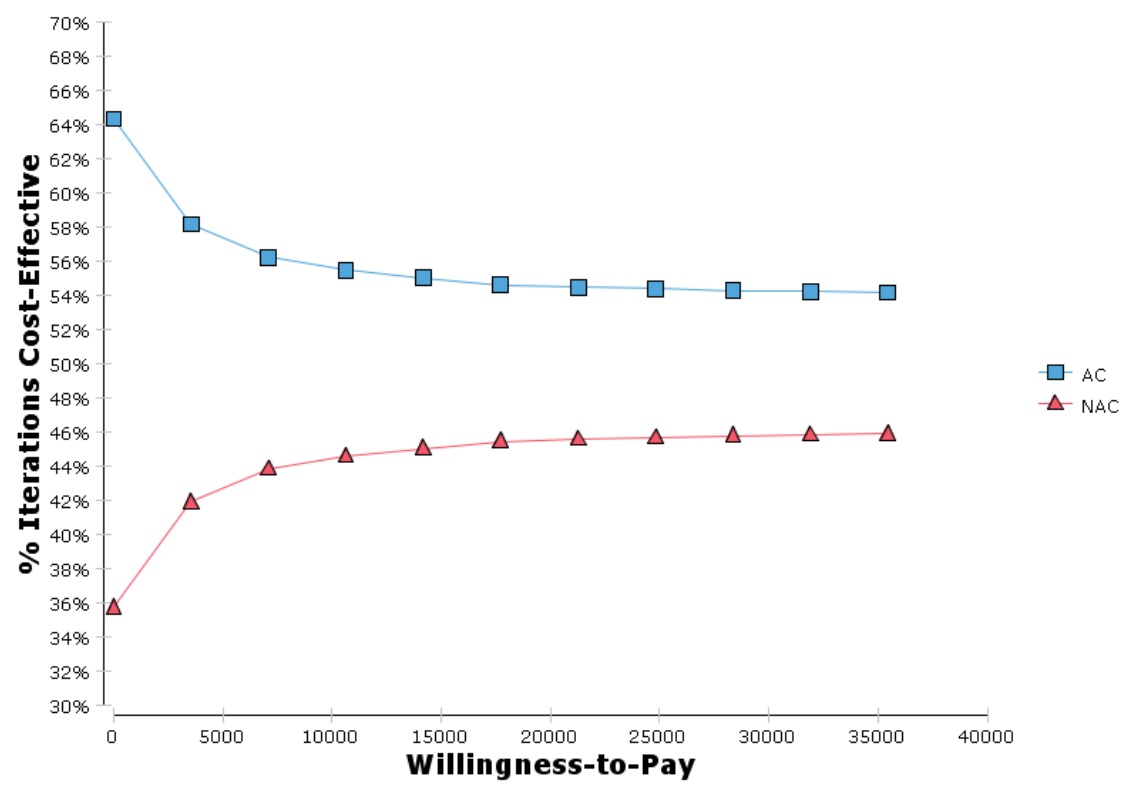




\section{Hosted file}

Table 1.pdf available at https://authorea.com/users/374783/articles/492221-costeffectiveness-of-neoadjuvant-versus-adjuvant-chemotherapy-for-ct2-4n0-1-non-small-celllung-cancer-patients-during-initial-treatment-phase

\section{Hosted file}

Table 2.pdf available at https://authorea.com/users/374783/articles/492221-costeffectiveness-of-neoadjuvant-versus-adjuvant-chemotherapy-for-ct2-4n0-1-non-small-celllung-cancer-patients-during-initial-treatment-phase

\section{Hosted file}

Table 3.pdf available at https://authorea.com/users/374783/articles/492221-costeffectiveness-of-neoadjuvant-versus-adjuvant-chemotherapy-for-ct2-4n0-1-non-small-celllung-cancer-patients-during-initial-treatment-phase

\section{Hosted file}

Table S 1.pdf available at https://authorea.com/users/374783/articles/492221-costeffectiveness-of-neoadjuvant-versus-adjuvant-chemotherapy-for-ct2-4n0-1-non-small-celllung-cancer-patients-during-initial-treatment-phase

\section{Hosted file}

Table S 2.pdf available at https://authorea.com/users/374783/articles/492221-costeffectiveness-of-neoadjuvant-versus-adjuvant-chemotherapy-for-ct2-4n0-1-non-small-celllung-cancer-patients-during-initial-treatment-phase 